\title{
PRODUÇÃO DE REVESTIMENTOS FUNCIONAIS COMPÓSITOS POR ELETRODEPOSIÇÃO.
}

\author{
L. G. LOPES ${ }^{1}$, D. ,T. G. de LIMA, A. V. C. BRAGA ${ }^{1}$, D. C. B. do LAGO$^{1}$ e L. F. de SENNA ${ }^{1}$ \\ ${ }^{1}$ Universidade do Estado do Rio de Janeiro, Departamento de Química Analítica \\ E-mail para contato: 1senna@uerj.br
}

\begin{abstract}
RESUMO - Revestimentos de cobre são usados em setores de engenharia devido a sua boa resistência à corrosão. Com o objetivo de melhorar as propriedades mecânicas do metal, partículas de óxidos são incorporadas ao revestimento. O desafio da obtenção desses materiais por eletrodeposição está em manter o óxido em suspensão durante o processo. Neste trabalho foram produzidos revestimentos compósitos $\mathrm{Cu}-\gamma-\mathrm{Al}_{2} \mathrm{O}_{3}$, utilizando um banho eletrolítico de $\mathrm{CuSO}_{4} 0,02 \mathrm{~mol} / \mathrm{L}, \mathrm{K}_{4} \mathrm{P}_{2} \mathrm{O}_{7} 0,90 \mathrm{~mol} / \mathrm{L}(\mathrm{pH}=8,14)$ no qual $20 \mathrm{~g} / \mathrm{L}$ de micropartículas de $\gamma-\mathrm{Al}_{2} \mathrm{O}_{3}$ foram adicionadas e mantidas em suspensão sob velocidade de agitação controlada, variando temperatura da solução, densidade de corrente e tempo de agitação prévia. As condições para a produção dos revestimentos compósitos foram selecionadas a partir das curvas de polarização catódicas, e arranjadas na forma de um planejamento composto fatorial $2^{4}$. Verificou-se que a variação do parâmetro densidade de corrente influenciou de forma positiva a eficiência de deposição.
\end{abstract}

\section{INTRODUÇÃO}

Revestimentos de cobre são largamente utilizados em setores de engenharia devido à boa condutividade elétrica e térmica, boa ductilidade e boa resistência à corrosão. Porém, esses revestimentos apresentam baixa resistência mecânica e de desgaste (ROBIN et al., 2009). Visando modificar as propriedades do cobre eletrolítico, vem sendo pesquisada a inclusão de partículas (cerâmica, polimérica ou metálica) ao revestimento. Estes tipos de materiais são denominados revestimentos funcionais compósitos e os mesmos têm por objetivo apresentar características específicas superiores às dos materiais originais. (ROBIN et al., 2010).

Uma das técnicas mais utilizadas para a produção de revestimentos funcionais compósitos é a técnica de codeposicão eletroquímica que consiste em incorporar partículas condutoras, semicondutoras ou não condutoras (adicionadas intencionalmente ao eletrólito) à matriz metálica durante o processo de eletrodeposição (OLIVEIRA et al., 2004; NOWAK et al., 2000; STANKOVIC, 1995). No entanto, o desafio da produção desses revestimentos está em manter as partículas em suspensão durante todo o processo de deposição. A grande dificuldade em propor mecanismos que expliquem o modo de incorporação destas partículas é a explicação física que justifique a dependência de alguns parâmetros que influenciam diretamente no processo de deposição, tais como a densidade de corrente, temperatura da solução, velocidade de agitação e tempo de agitação prévia (GUGLIELMI, 1972; CELLIS et al., 1987; MALFATTI, 2004; (NORKUS, KEPENIENE, 


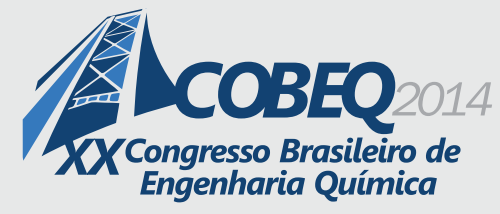

STALNIONIENE, 2012).

$\mathrm{O}$ presente estudo visa a produção de revestimentos compósitos de $\mathrm{Cu}-\gamma-\mathrm{Al}_{2} \mathrm{O}_{3}$ por codeposição eletroquímica, empregando um planejamento composto fatorial $2^{4}$ para a otimização de parâmetros de deposição (temperatura da solução, densidade de corrente, velocidade de agitação e tempo de agitação prévio).O objetivo é contribuir para a melhor compreensão dos processos de deposição que envolvem a eletrodeposição de codepósitos Me/partícula cerâmica.

\section{MATERIAIS E MÉTODOS}

Ensaios de polarização potenciodinâmica foram realizados empregando uma célula de três eletrodos, onde o eletrodo de trabalho usado foi um disco de aço ao carbono AISI 1028, com área exposta de $7,85 \times 10^{-5} \mathrm{~m}^{2}$ imerso em uma solução composta de $\mathrm{K}_{4} \mathrm{P}_{2} \mathrm{O}_{7} 0,90 \mathrm{~mol} / \mathrm{L}, \mathrm{CuSO}_{4} 0,02$ $\mathrm{mol} / \mathrm{L}(\mathrm{pH}=8,14)$, com adição de $20 \mathrm{~g} / \mathrm{L}$ de $\gamma-\mathrm{Al}_{2} \mathrm{O}_{3}$. Como contra eletrodo, foi utilizada uma placa de cobre e o potencial foi lido contra um eletrodo de referência de sulfato mercuroso saturado (ESS). Os experimentos foram conduzidos a diferentes temperaturas $\left(25^{\circ} \mathrm{C}, 35^{\circ} \mathrm{C}, 45^{\circ} \mathrm{C}\right.$ e $\left.55^{\circ} \mathrm{C}\right)$, diferentes velocidades de agitação ( $800 \mathrm{rpm}, 1000 \mathrm{rpm}, 1200 \mathrm{rpm}$ e $1400 \mathrm{rpm}$ ) e diferentes tempos de agitação prévia ( $1 \mathrm{~h}, 2 \mathrm{~h}, 3 \mathrm{~h}, 4 \mathrm{~h}, 5 \mathrm{~h}$ e $6 \mathrm{~h}$ ). A partir das curvas de polarização foram selecionados valores de densidade de corrente $(\boldsymbol{I})$, tempo de agitação prévia $(\boldsymbol{t})$ e velocidade de agitação $(\boldsymbol{A})$ e temperatura da solução $(\boldsymbol{T})$ objetivando verificar as melhores condições para a produção dos revestimentos compósitos.

Os ensaios de eletrodeposição foram realizados a partir do planejamento composto fatorial $2^{4}$, apresentado na Tabela 1, avaliando-se quantitativamente a influência das variáveis independentes (densidade de corrente $\boldsymbol{I}$, tempo de agitação prévia $\boldsymbol{t}$, velocidade de agitação $\boldsymbol{A}$ e temperatura da solução $\boldsymbol{T})$ sobre a eficiência de corrente catódica $\left(\boldsymbol{E}_{\boldsymbol{f}}\right)$, teores do cobre $(\boldsymbol{\%} \mathbf{m} \mathbf{m} \boldsymbol{C u})$ e de $\gamma$-Alumina $\left(\% \mathbf{m} / \mathbf{m} \boldsymbol{\gamma A l}_{2} \mathrm{O}_{3}\right.$ ) no revestimento compósito. Após os ensaios de eletrodeposição, os revestimentos foram solubilizados em $\mathrm{HNO}_{3} 65 \% \mathrm{v} / \mathrm{v}$, sendo o cobre e o alumínio posteriormente analisados por espectrometria de emissão ótica por plasma indutivamente acoplado (ICP-OES). A partir destes dados foram obtidas as massas depositadas para cada um dos componentes metálicos dos revestimentos $(\mathrm{Cu}$ e Al), bem como a massa total do depósito e consequentemente, a massa de $\gamma-\mathrm{Al}_{2} \mathrm{O}_{3}$.

Para a análise da estatística dos dados experimentais foi usado o software STATISTICA for Windows, versão 7.0. A Equação 1 representa o modelo correspondente a superfície quadrática completa entre as respostas e os fatores.

$$
\hat{y}=b_{a}+b_{1} x_{1}+b_{2} x_{2}+b_{3} x_{3}+b_{11} x_{1}^{2}+b_{22} x_{2}^{2}+b_{33} x_{3}^{2}+b_{12} x_{1} x_{2}+b_{13} x_{1} x_{3}+b_{23} x_{2} x_{3}
$$

onde $\hat{y}$ é a variável predita $\left(\boldsymbol{E}_{\boldsymbol{f}}, \boldsymbol{\%} / \boldsymbol{m} \boldsymbol{C u}\right.$ e $\left.\% \boldsymbol{m} / \boldsymbol{m} \boldsymbol{\gamma}-\mathrm{Al}_{2} \mathrm{O}_{3}\right), \mathrm{x}_{1}$ corresponde à $\mathrm{T}, \mathrm{x}_{2}$ corresponde à $\boldsymbol{A}, \mathrm{x}_{3}$ representa $\boldsymbol{t}$ e $\mathrm{x}_{4}$ representa $\boldsymbol{I}, \mathrm{x}_{\mathrm{i}} \mathrm{x}_{\mathrm{j}}$ é o termo da interação entre dois dos parâmetros estudados, $b_{i}$ são coeficientes da equação. Tal equação foi aplicada para todas as variáveis, utilizando, com base no teste $\mathrm{t}(95 \%$ de confiança), todos os efeitos que exerçam influência significativa $(\mathrm{p}<$ $0,05)$. 
Tabela 1 - Matriz do planejamento linear $2^{4}$ e valores reais empregados no planejamento

\begin{tabular}{|c|c|c|c|c|c|c|c|c|}
\hline \multirow{2}{*}{ Ensaios } & \multicolumn{3}{|c|}{ Variável Codificada } & \multicolumn{5}{c|}{ Variáveis reais } \\
\cline { 2 - 9 } & $\mathrm{X}_{1}$ & $\mathrm{X}_{2}$ & $\mathrm{X}_{3}$ & $\mathrm{X}_{4}$ & $\mathrm{~T}\left({ }^{\circ} \mathrm{C}\right)$ & $\mathrm{A}(\mathrm{rpm})$ & $\mathrm{t}(\mathrm{h})$ & $\mathrm{I}\left(\mathrm{A} / \mathrm{m}^{2}\right)$ \\
\hline $\mathbf{1}$ & -1 & -1 & -1 & -1 & 30 & 1000 & 2 & 7 \\
\hline $\mathbf{2}$ & 1 & -1 & -1 & -1 & 50 & 1000 & 2 & 7 \\
\hline $\mathbf{3}$ & -1 & 1 & -1 & -1 & 30 & 1300 & 2 & 7 \\
\hline $\mathbf{4}$ & 1 & 1 & -1 & -1 & 50 & 1300 & 2 & 7 \\
\hline $\mathbf{5}$ & -1 & -1 & 1 & -1 & 30 & 1000 & 6 & 7 \\
\hline $\mathbf{6}$ & 1 & -1 & 1 & -1 & 50 & 1000 & 6 & 7 \\
\hline $\mathbf{7}$ & -1 & 1 & 1 & -1 & 30 & 1300 & 6 & 7 \\
\hline $\mathbf{8}$ & 1 & 1 & 1 & -1 & 50 & 1300 & 6 & 7 \\
\hline $\mathbf{9}$ & -1 & -1 & -1 & 1 & 30 & 1000 & 2 & 21 \\
\hline $\mathbf{1 0}$ & 1 & -1 & -1 & 1 & 50 & 1000 & 2 & 21 \\
\hline $\mathbf{1 1}$ & -1 & 1 & -1 & 1 & 30 & 1300 & 2 & 21 \\
\hline $\mathbf{1 2}$ & 1 & 1 & -1 & 1 & 50 & 1300 & 2 & 21 \\
\hline $\mathbf{1 3}$ & -1 & -1 & 1 & 1 & 30 & 1000 & 6 & 21 \\
\hline $\mathbf{1 4}$ & 1 & -1 & 1 & 1 & 50 & 1000 & 6 & 21 \\
\hline $\mathbf{1 5}$ & -1 & 1 & 1 & 1 & 30 & 1300 & 6 & 21 \\
\hline $\mathbf{1 6}$ & 1 & 1 & 1 & 1 & 50 & 1300 & 6 & 21 \\
\hline $\mathbf{1 7}$ & 0 & 0 & 0 & 0 & 40 & 1150 & 4 & 14 \\
\hline $\mathbf{1 8}$ & 0 & 0 & 0 & 0 & 40 & 1150 & 4 & 14 \\
\hline $\mathbf{1 9}$ & 0 & 0 & 0 & 0 & 40 & 1150 & 4 & 14 \\
\hline
\end{tabular}

\section{RESULTADOS E DISCUSSÃO}

\subsection{Curvas de Polarização Potenciodinâmica}

A Figura 1 mostra alguns exemplo das curvas de polarização do aço levantadas no meio contendo $\mathrm{Cu}(\mathrm{II})-\mathrm{K}_{2} \mathrm{P}_{2} \mathrm{O}_{7}+$ partículas de $\gamma$-alumina, a partir de variações dos parâmetros de deposição estudados. As curvas obtidas em $\boldsymbol{A}=800 \mathrm{rpm}$ e em $\boldsymbol{T}=25^{\circ} \mathrm{C}$ (Figura 1A) seguem o mesmo perfil independente de $\boldsymbol{t}$, sugerindo que esse parâmetro não interfere tanto nos potenciais de circuito aberto quanto nos potenciais ao longo da curva, para os valores de $\boldsymbol{A}$ e $\boldsymbol{T}$ estudados. Contudo, com o aumento de $\boldsymbol{A}$ (Figura 1B), no memo valor de $\boldsymbol{T}$, é possível verificar uma despolarização do substrato em condições de 4 horas de de agitação prévia, na região em que a deposição do cobre é controlada por transferência de massa. Nesse caso, as condições de $\boldsymbol{A}$ favorecem que o parâmetro $\boldsymbol{t}$ possa afetar a deposição do cobre e, consequentemente, o carreamento das partículas de alumina para a superfície do eletrodo. $\mathrm{O}$ aumento da velocidade de agitação do eletrólito é considerado um fator fundamental para a estabilização das partículas em suspensão, principalmente em casos onde o seu valor absoluto de potencial zeta é baixo (GARCÍA-LECINA, et al., 2009). 


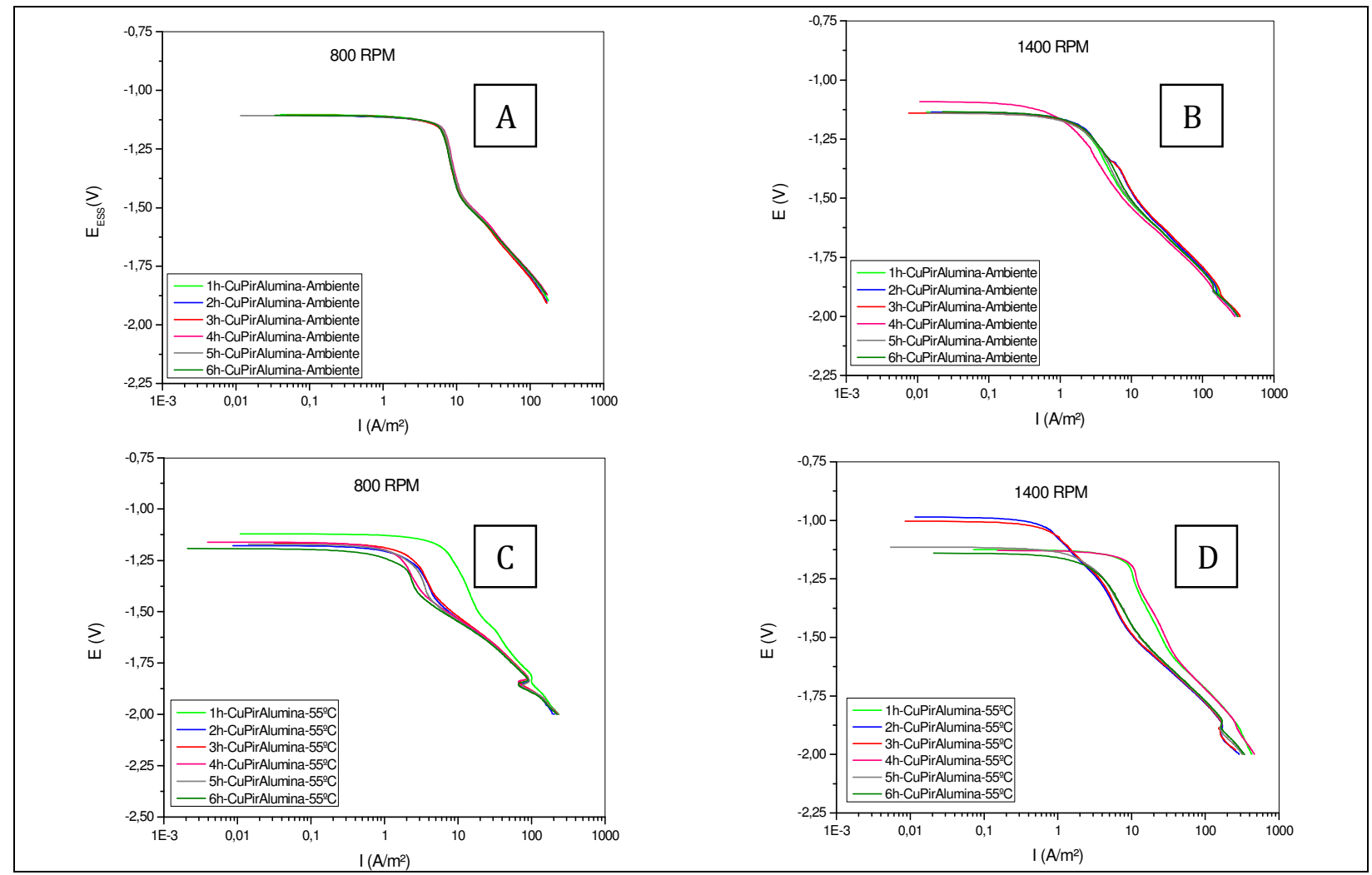

Figura 1 - Curvas de polarização potenciodinâmica do banho de CuSO4 0,02 mol/L, K4P2O7 0,90 mol/L e $20 \mathrm{~g} / \mathrm{L}$ de $\gamma$-Al2O3 sob velocidade de agitação e temperatura de (A) $800 \mathrm{rpm}, 25^{\circ} \mathrm{C}$, (B) $1400 \mathrm{rpm}, 25^{\circ} \mathrm{C}$, (C) $800 \mathrm{rpm}, 55^{\circ} \mathrm{C}$ e (D) $1400 \mathrm{rpm}, 55^{\circ} \mathrm{C}$. Velocidade de varredura $=1 \mathrm{mV} / \mathrm{s}$.

É possível notar uma despolarização do substrato nas curvas de polarização obtidas na velocidade de agitação de $1400 \mathrm{rpm}$ e temperatura de $55^{\circ} \mathrm{C}$, para as condições de 2 e 3 horas de agitação prévia (Figura 1D). Este mesmo comportamento é visualizado nas curvas obtidas na velocidade de agitação de $800 \mathrm{rpm}$ e temperatura $55^{\circ} \mathrm{C}$ (Figura 1C), onde o substrato sofreu uma despolarização no ensaio com $1 \mathrm{~h}$ de agitação prévia. Esses resultados indicam que poderia haver maior facilidade na redução dos íons cobre em menores tempos de agitação prévios na temperatura acima da temperatura ambiente (NORKUS, KEPENIENE, STALNIONIENE, 2012).

\subsection{Eletrodeposição dos Revestimentos Compósitos $\mathrm{Cu}-\gamma-\mathrm{Al}_{2} \mathrm{O}_{3}$}

Eficiência de corrente catódica $\left(\mathrm{E}_{\mathrm{f}}\right)$ : A Figura 2 apresenta o diagrama de Pareto para a variável $\boldsymbol{E}_{\boldsymbol{f}}$ com base no planejamento apresentado na Tabela 1. A magnitude de cada efeito é apresentada em colunas e a linha transversal às colunas, correspondente ao valor de $p=0,05$, indica o quão grande deve ser $o$ efeito para ter significado estatístico. Com 95\% de confiança foi observado que o parâmetro densidade de corrente influenciou $\boldsymbol{E}_{f}$, conforme apresentado na Equação 2. Isso significa que os valores de eficiência de corrente catódica são independentes de $\boldsymbol{A}, \boldsymbol{T}$ e $\boldsymbol{t}$ e dependentes de $\boldsymbol{I}$. 


$$
\boldsymbol{E}_{f}=83,21+7,87 \boldsymbol{I}
$$

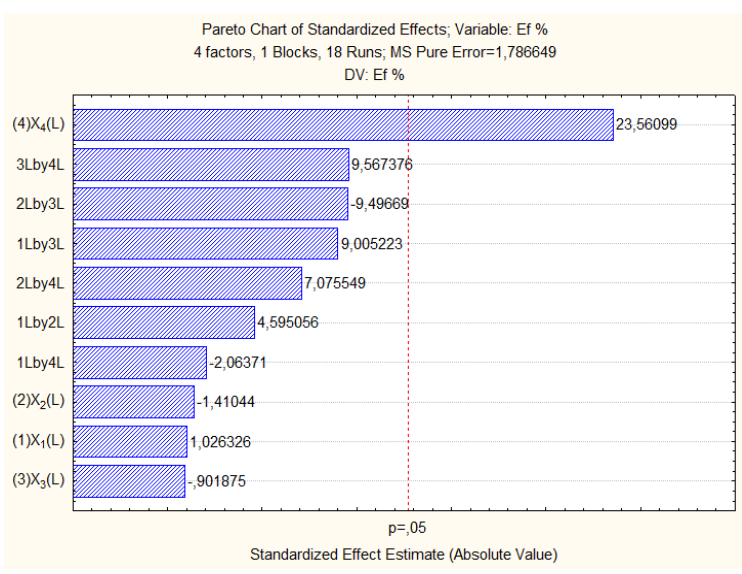

Figura 2 - Diagrama de Pareto dos efeitos do planejamento fatorial. Variável: Eficiência de Corrente Catódica no revestimento (Ef).

Na Figura 3 é possível observar as tendências nas superfícies de resposta da influência dos parâmetros estudados sobre a variável analisada para a produção do revestimento compósito. É possível notar que o parâmetro mais relevante na variável eficiência de corrente catódica $\left(E_{f}\right)$ é a $\boldsymbol{I}$. Independente da faixa do outro parâmetro, quanto maior $\boldsymbol{I}(\mathrm{p}<0,03)$, maior é $\boldsymbol{E}_{\boldsymbol{f}}$.

Analisando as tendências não significativas, na Figura 3B verifica-se que o aumento ou o decréscimo simultaneo de $\boldsymbol{A}$ e $\boldsymbol{T}$ aumentam os valores de $\boldsymbol{E}_{f}$. Já a Figura $3 \mathrm{C}$ apresenta melhores resultados quando ambos os parâmetros $\boldsymbol{T}$ e $\boldsymbol{t}$ são extremos. É possível observar que com o aumento ou redução da temperatura e do tempo de agitação prévio ocorre o aumento da eficiência de deposição, uma vez que a temperatura da solução aumenta a deposição do cobre e o tempo de agitação prévio estabiliza a partícula de $\gamma$-Alumina, favorecendo também sua deposição (LIMA, 2013; AGARWALA et al., 2003). É possível notar ainda que tanto o aumento de $\boldsymbol{t}$ e o simultâneo decréscimo de $\boldsymbol{A}$, quanto o aumento nas direções inversas, poderiam causar um aumento em $\boldsymbol{E}_{\boldsymbol{f}}$ (Figura 3E). Por outro lado, um aumento ou decréscimo conjunto destes dois parâmetros prejudicaria a deposição do material compósito, tendendo a diminuir sua $\boldsymbol{E}_{\boldsymbol{f}}$. Aparentemente, os resultados sugerem que, para um mesmo valor de $\boldsymbol{I}$ e $\boldsymbol{T}$, as melhores condições para elevar a $\boldsymbol{E}_{\boldsymbol{f}}$ destes revestimentos seria variação destes dois parâmetros em direções contrárias (LIMA, 2013).

Teores de Cobre $(\% \mathrm{~m} / \mathrm{m} \mathrm{Cu})$ e de $\gamma$-Alumina $\left(\% \mathrm{~m} / \mathrm{m} \gamma \boldsymbol{A l}_{2} \underline{\boldsymbol{O}_{3}}\right)$ no Revestimento Compósito: Foi observado que nenhum dos parâmetros estudados influenciou estas variáveis, exceto o valor médio $\left(\mathrm{b}_{0}\right)$. Isso significa que tanto $\% \mathrm{~m} / \boldsymbol{m} \mathbf{C u}$ quanto $\% \mathbf{m} / \boldsymbol{m} \boldsymbol{\gamma} \boldsymbol{A l}_{2} \mathrm{O}_{3}$ são independentes de $I, A, t \boldsymbol{e} T$. Além disso, foi verificado que as influências (não significativas) de ambas as variáveis combinavam-se e tendiam em sentido inverso. Assim, as superfícies de resposta para a variável $\% \mathbf{m} / \boldsymbol{m} \boldsymbol{C u}$ (Figura 4) é apresentada como exemplo para discussão dessas influências. 


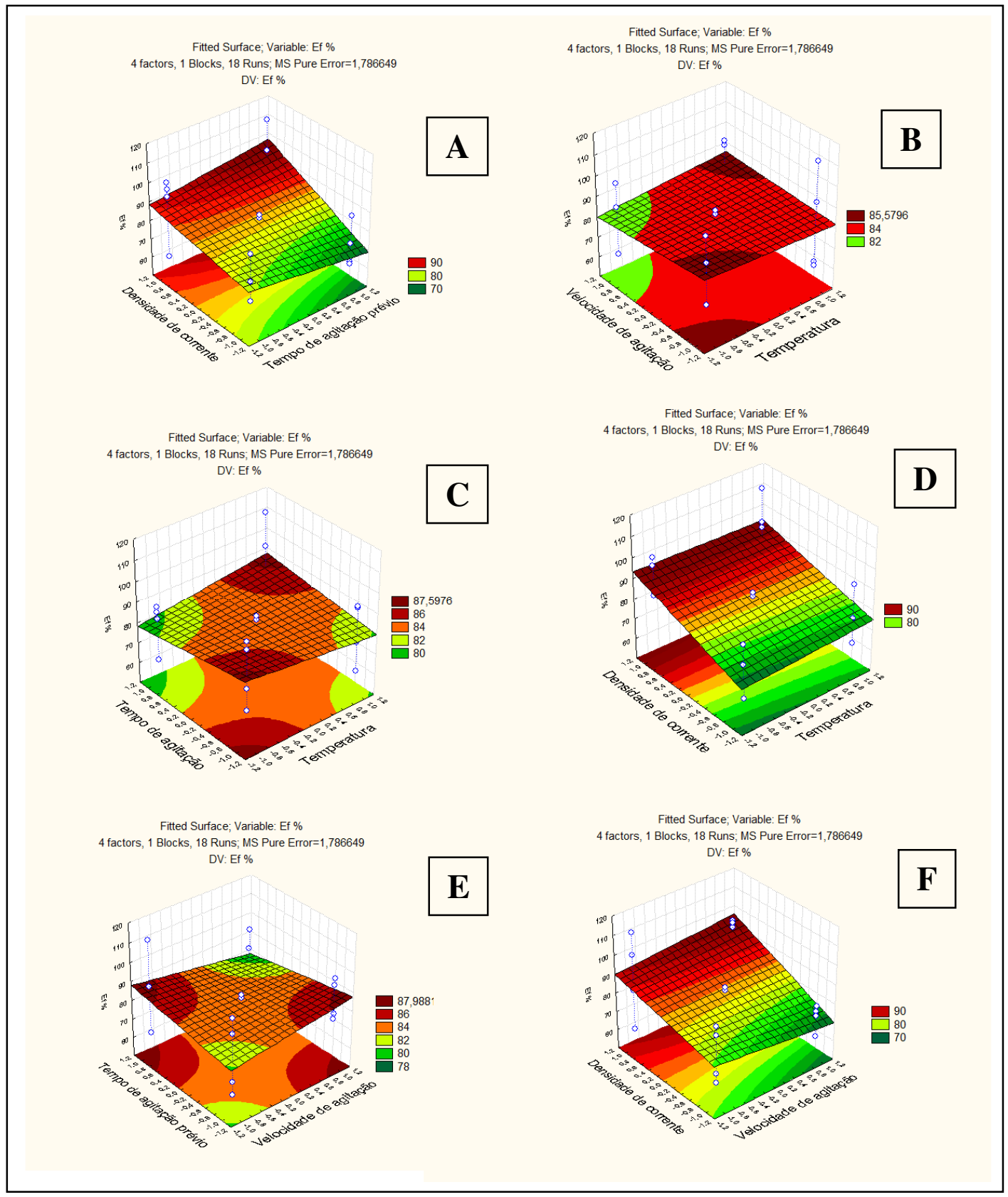

Figura 3 - (A) Superfície de resposta da interação entre $\boldsymbol{I}$ e $\boldsymbol{t}$; (B) Superfície de resposta da interação entre $\boldsymbol{A}$ e $\boldsymbol{T}$; (C) Superfície de resposta da interação entre $\boldsymbol{t}$ e $\boldsymbol{T}$. (D) Superfície de resposta da interação entre $\boldsymbol{I}$ e $\boldsymbol{T}$ (E) Superfície de resposta da interação entre $\boldsymbol{t}$ e $\boldsymbol{A}$. (F) Superfície de resposta da interação entre $\boldsymbol{I}$ e $\boldsymbol{A}$. Variável: Eficiência de Corrente Catódica no revestimento $\left(\boldsymbol{E}_{f}\right)$. 


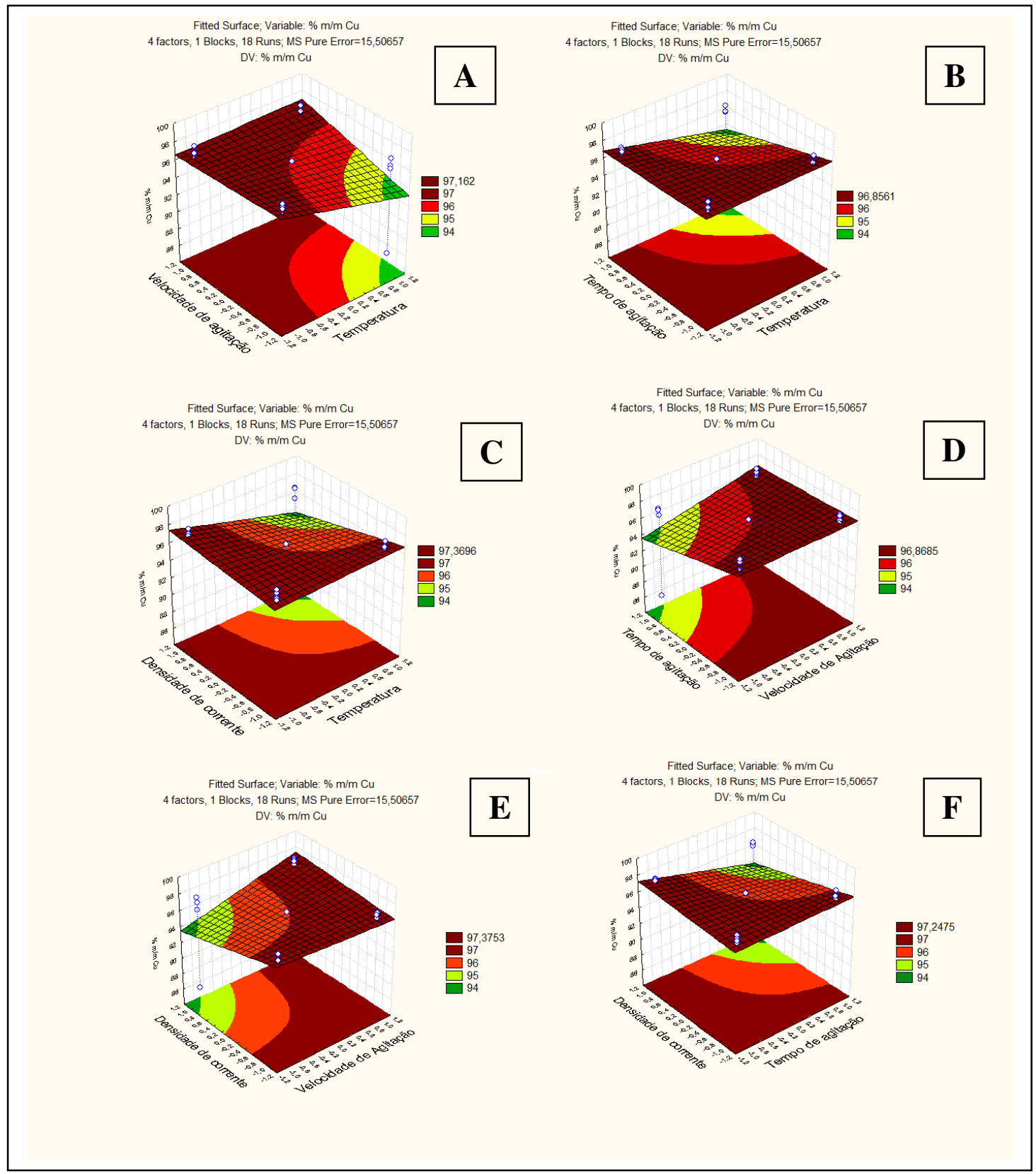

Figura 4- (A) Superfície de resposta da interação entre $\boldsymbol{A}$ e $\boldsymbol{T}$; (B) Superfície de resposta da interação entre $\boldsymbol{t}$ e $\boldsymbol{T}$. (C) Superfície de resposta da interação entre $\boldsymbol{I}$ e $\boldsymbol{T}$ (D) Superfície de resposta da interação entre $\boldsymbol{t}$ e $\boldsymbol{A}$. (E) Superfície de resposta da interação entre $\boldsymbol{I}$ e $\boldsymbol{A}$. (F) Superfície de resposta da interação entre $\boldsymbol{I}$ e $\boldsymbol{t}$; Variável: Teor de Cobre no Revestimento Compósito $(\% \mathrm{~m} / \mathrm{m} \mathrm{Cu})$.

Em função de ser um metal com características nobres, a redução do $\mathrm{Cu}$ tende a ocorrer em menores valores de $\boldsymbol{I}$ (potenciais menos negativos) (LIMA, 2013). Analisando as superfícies de 


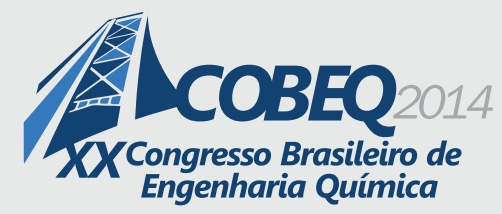

19 a 22 de outubro de 2014

Florianópolis/SC

resposta com o parâmetro densidade de corrente, nota-se que quando $I$ assume baixos valores (Figuras 4C, 4D e 4F), independente do outro parâmetro $\boldsymbol{A}, \boldsymbol{T}$ e $\boldsymbol{t}$, a deposição de Cu é favorecida. Logo, o decréscimo de $\boldsymbol{I}$ favorece a depoisção de $\boldsymbol{\gamma}$-Alumina. Por outro lado, o aumento de $\boldsymbol{A}$ também favorece um aumento do $\% \boldsymbol{m} / \boldsymbol{m} \boldsymbol{C u}$ no revestimento, principalment quando associado com decréscimo de $\boldsymbol{T}$ e $\boldsymbol{t}$ (Figuras 4A e 4D). Elevados valores de $\boldsymbol{A}$ são, geralmente, condições que favorecem a deposição de cobre (LIMA, 2013). Por sua vez, a diminuição de $\boldsymbol{t}$ desestabiliza a suspensão da $\gamma$-Alumina, também favorecendo a deposição do cobre. Assim, por analogia, condições inversas a estas são favoráveis à deposição de $\boldsymbol{\gamma}$-Alumina. Porém, esperava-se que o aumento de $\boldsymbol{T}$ favorecesse o aumento do $\mathbf{\%} \mathbf{m} / \boldsymbol{m}$ $\boldsymbol{C u}$ no revestimento por aumentar a difusão. Mas o que foi verificado é que um aumento desta variável aumentou o teor de $\gamma$-Alumina no depósito.

\section{CONCLUSÃO}

Nas condições estudadas, maiores valores de $\boldsymbol{I}$ são condições que favorecem significativamente a elevação de $\boldsymbol{E} \boldsymbol{f}$ em banhos de pirofosfato, independente dos outros parâmetros estudados. Embora não haja influência significativa, esta condição parece favorecer o aumento do teor de $\gamma-\mathrm{Al}_{2} \mathrm{O}_{3}$ no revestimento.

\section{REFERENCIAS}

CELIS, J.P.; ROOS, J.R.; BUELENS, C. A mathematical model for the electrolyte codeposition of particles with a metallic matrix. Journal of the Electrochemical Society, 1987 v. 134, p. 1402 - 1408 , 1987.

ERAUZKIN, E.A.; STILLER, K.; DUNLOP, G.L.; COUPER, M.J. The precipitation and sequence in Al-Mg-Si alloys. Actra Materialia. v. 46, p. 3893 - 3904, 1998.

GUGLIELMI, N. Kinectics of the deposition of inert particles from electrolyte baths. J. Electrochem. Soc v.119, p.1009 - 1012, 1972.

MAFALTTI, C.F. Elaboração e Caracterização de Nanocompósitos Ni-P-SiC Eletrodepositados. 147p. Tese (Doutorado em Engenharia) - Universidade Federal do Rio Grande do Sul, Porto Alegre, RS. 2004.

NORKUS, E.; KEPENIENE, V.; STALNIONIENE, I.; Application of environment-friendly ligands for alkaline electroless copper plating systems: A comparative study of electroless copper deposition using D-, L- and DL-tartrate as Cu(II) ligands. chemija. Lietuvos mokslu akademija, 2012vol. 23. No. 3. P. 155-170.

NOWAK, P.; SOCHA, R.P.; KAISHEVA, M.; FRANSAER, J.; CELIS, J-P.; STOINOV, Z. Electrochemical investigation of the codeposition of $\mathrm{SiC}$ and $\mathrm{SiO}_{2}$ particles with nickel. Journal of Applied Electrochemistry. v. 30, p. $429-437,2000$.

OLIVEIRA, R.S.; PINHEIRO, M.A.S. Caracterização de Materiais Compósitos. Trabalho apresentado na XI CREEM, Instituto Politécnico, Nova Friburgo, RJ, 30 de agosto a 3 setembro, 2004.

ROBIN, A.; SANTANA, J.C.P.; SARTORI, A.F. Characterization of copper-silicon nitride composite electrocoatings. Journal of Applied Electrochemistry, v.40, p. 507- 513, 2010.

STANKOVIC, V.D.; GOJO, M. Electrodeposited composite coatings of copper with inert, semiconductive and conductive particles. Surface and Coatings Techonology, v.81, p. 225-232, 1996. 